\title{
ANALYSIS OF THE REVERSE JET INFLUENCE ON PARTICLE INGESTION AT THE ENGINE INLET
}

\section{S. Yu. Krasheninnikov and D. E. Pudovikov}

Central Institute of Aviation motors (CIAM)

2 Aviamotornaya Str., Moscow 111116, Russia

The reverse mode of an operating near-ground jet engine is considered. The air flow and particle trajectories under the engine intake are calculated. On the base of numerical and theoretical analysis of the air flow and particle trajectories, some conclusions about reducing the probability of transportation of large particles to the engine are drawn.

\section{INTRODUCTION}

The low underwing engine location, which is typical for modern civil airplane configurations, is of the reason for a number of unfavorable factors. Increasing probability of transportation of large particles to the engine inlet is one of them. Among the reasons for large particle scattering and suction in engine are the landing gear motion on the ground, the action of a reverse jet, and the powerful vortex flow formed due to engine operation near the surface.

The regime of trust reversing, which is used for airplane braking on the ground, is one of critical regimes for safety and reliability of aviation engines. During the thrust reverse activities, the probability of suction of large particles into the engine, which leads to blade damage, sufficiently increases. Moreover, suction of the exhaust gas to the engine becomes possible, which leads to increased nonuniformity of flow parameters.

The particle motion can be caused by some kind of the mechanical action or by the action of the vortex flow and reverse jet on the near-surface engine operation regime. It is necessary to distinguish these reasons.

The vortex flow has a property of particle accumulation in the near-surface region of its influence. As a result, the flow lifts particles from this region to the engine inlet. The size of particles lifted by the vortex flow depends on the rotation motion intensity. The particle lifting process is very difficult and 
depends on a number of different effects: particles shifting and transporting by the flow, interaction between particles, etc. In [1,2], it was shown that the size of particles lifted by the vortex flow can be ten times greater than that without the vortex.

The interaction of a reverse jet and a counterflow also generates conditions for particle accumulation in a zone of flow turning. In accordance with $[1,2]$, this leads to conditions for arising of suction of large particles. The analysis of the interaction of reverse jets, which extend along the surface and are turned by the counterflow, with the flow at the engine input demonstrated that conditions for suction of large particles into the engine are significantly enhanced.

\section{PARTICLE ACCUMULATION IN THE VORTEX}

A detailed analysis of the process of particle accumulation in the vortex was performed in $[1,2]$. Therefore, in this section, only the main results, which are important for problems of the reverse jet influence on suction of large particles to the engine inlet, will be considered.

For particles lifted by the flow, the drag force $f$ should be greater than the gravity force $P$. The maximum diameter of lifted particles can be estimated from the equilibrium conditions $P=f$ as

$$
d=\frac{3 \rho}{4 \rho_{p} g} u^{2}
$$

where $u$ is the flow velocity; $\rho_{p}$ is the particle density; and $\rho$ is the density of the moving ambient medium (it is accepted that Stokes factor of the drag is $C_{x}=1$ ).

For the further analysis of the distribution of velocity components, it is convenient to use the velocity $U$, which is induced in the near-surface layer by the intake duct with a radius $R_{i}$. In the free space, the level of velocities at a distance $H$ from the intake channel can be estimated by the equation

$$
\frac{U}{u_{i}}=\left(\frac{1}{2} \ldots \frac{1}{4}\right) \frac{R_{i}^{2}}{H^{2}}
$$

The coefficient $1 / 2$ corresponds to small values of the ratio $H / R_{i}$, and the coefficient $1 / 4$ corresponds to $H / R_{i} \rightarrow \infty$. Here, $u_{i}$ is the velocity of the inlet flow in the duct. In the case where the surface is located at the distance $H$, the values of $U$ can be used for evaluation of the induced near-surface velocity.

A numerical analysis of the near-surface flow during engine operation [1-3] demonstrates that the velocities in this region are not greater than the induced velocity $U$. The values of the vertical velocity increase from zero and reach the value $V_{z} \approx U$ at the height approximately $R_{i}$ above the surface. Therefore, the 


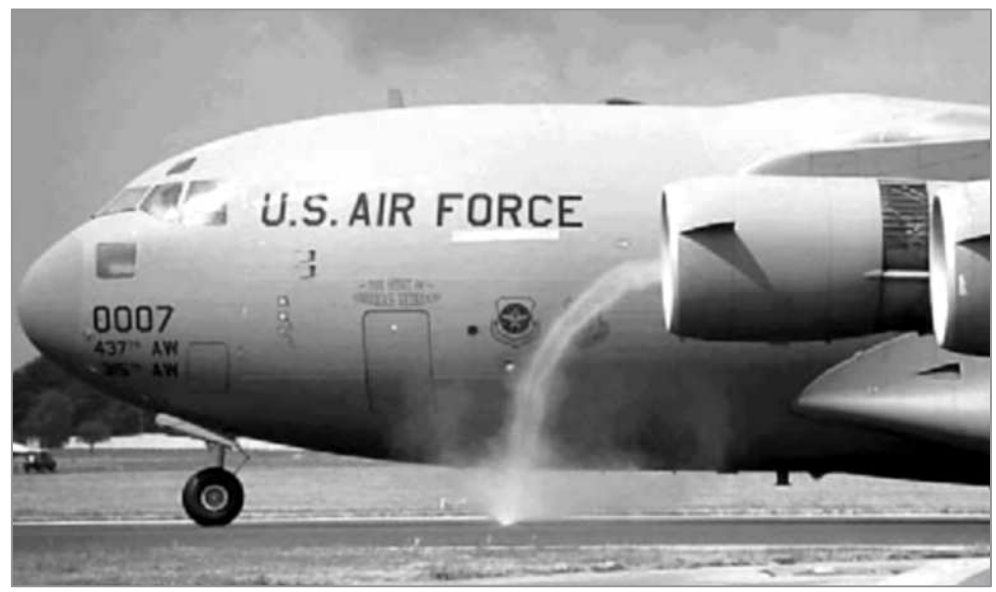

Figure 1 Photo of the vortex flow at the engine inlet

air motion in the near-surface region cannot move large particles. Accumulation of small particles in the vortex region is needed for lifting large particles. This creates a moving medium whose medium density $\rho$ is sufficiently greater than that of air. The particle accumulation time was evaluated in [1]. For real conditions, it is approximately several seconds. Small particles create vortex visualization effects demonstrated in Fig. 1.

When the airplane moves, the vortex flow at the engine input does not disappear and moves with it. It is confirmed by the corresponding numerical calculations. However, the conditions for particle accumulation at the vortex base are disturbed and an illusion of vortex disappearance at the engine input during airplane motion with velocities greater than several meters per second arises. In [1-3], numerical calculations of the flow were performed, which demonstrated that the vortex flow intensity decreases only by several percent for real airplane engine configurations at counterflow velocities of $50 \mathrm{~m} / \mathrm{s}$. It shows that the vortex flow at the engine inlet exists in all modes of motions during airplane landing.

\section{REVERSE JET PROPAGATION}

\subsection{Problem Formulation}

In the scope of this section, jet propagation from the thrust reversing device along the surface and its incidence onto the air intake are considered. Therefore, aspects associated with features of jet generation, leakages, and thrust reverse 


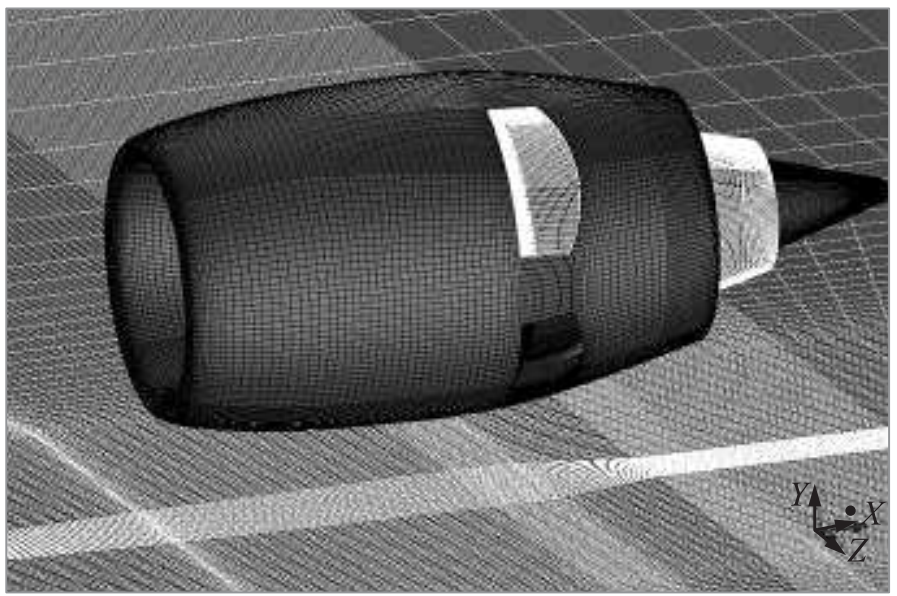

(a)

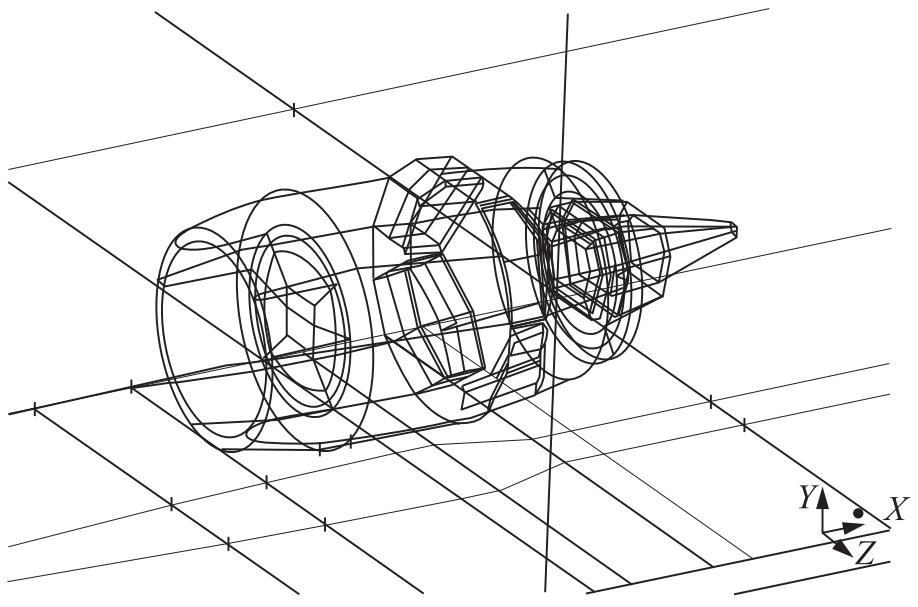

(b)

Figure 2 External view of the pod and model of the thrust reverse device $(a)$ and topology of the calculated area on the pod and ground surfaces $(b)$

device structure were not considered. The model of a pod for the engine with a bypath ratio approximately equal to 10 was used for calculations (Fig. 2).

Experience of numerical simulations of model thrust reverse devices demonstrated that the geometric details of devices affect the flow parameters inside them and the characteristics of the initial part of the reversing jet. However, jet propagation in the flow is practically independent of this geometry and de- 
Table 1 Engine operation parameters

\begin{tabular}{cccccc}
\hline$G_{2}, \mathrm{~kg} / \mathrm{s}$ & $P_{2}^{*}, \mathrm{~kg} / \mathrm{cm}^{2}$ & $T_{2}^{*}, \mathrm{~K}$ & $G_{1}, \mathrm{~kg} / \mathrm{s}$ & $P_{1}^{*}, \mathrm{~kg} / \mathrm{cm}^{2}$ & $T_{1}^{*}, \mathrm{~K}$ \\
\hline 400 & 1.5 & 320 & 40 & 1.1 & 800 \\
\hline
\end{tabular}

pends only on the angle of the exit flow and its total parameters. Therefore, for saving numerical resources, the cavity in the pod surface was used instead of the detailed description of the thrust reverse device. The spatial orientation of the cavity corresponded to the orientation of the initial part of the reverse jet and its size corresponded to the size of the reverse door or cascade. At the cavity bottom, the total parameters of jet were set. The losses inside the thrust reverse device were not considered in this work. Therefore, total parameters of the reverse jet corresponded to the total parameters in the secondary flow of the engine. This approach does not give the opportunity to investigate the problem of the jet adhering to the pod, the efficiency of the selected thrust reverse device, etc. Nevertheless, this approach is applicable for the reverse jet propagation description.

The static pressure was set inside the air inlet in the cross section corresponding to the fan inlet plane. Its value was selected from the condition of prescribed mass flow rates through the air inlet.

The flow parameters are summarized in Table 1 . Here, $G$ is the mass flow rate, $P^{*}$ and $T^{*}$ are the total pressure and temperature. The subscripts 1 and 2 refer to the primary and secondary flow parameters, respectively. The mass flow rate through the air intake was set as the sum $G_{1}+G_{2}$.

It was demonstrated $[1,2]$ that the exhaust jet affects the flow parameters ahead of the air intake. Therefore, the used model included the nozzle duct for exhaust jet modeling in order to take this effect into account. The exhaust jet flow parameters were set in accordance with the primary flow parameters.

The sizes of the calculation domain were selected to eliminate the influence of the boundary on the flow near the engine. The distances between the pod and the front and side boundaries were 30 inlet diameters, and the distance between the pod and the backside was 50 inlet diameters. The distance between the engine and the downside corresponds to the height of engine location.

The Cobra in-house computational fluid dynamics (CFD) solver was used. This program was developed for solving aerodynamic problems on the base of the Reynolds-averaged Navier-Stokes (RANS) technology (on the base of numerical integration of the Reynolds equations closed by the $\nu_{t}-90$ turbulence model). This program uses a multiblock mesh. Figure $2 b$ demonstrates the topology of the mesh used for calculations. This mesh is refined toward the pod and ground surfaces. The calculated with the size of the first near-surface cell value of $y^{+}$ was equal to 10 for the pod and 1 for the ground. For significant reduction of the 


\section{$T / \mathrm{K}$}

$\begin{array}{llllll}273 & 278 & 283 & 288 & 293 & 298\end{array}$

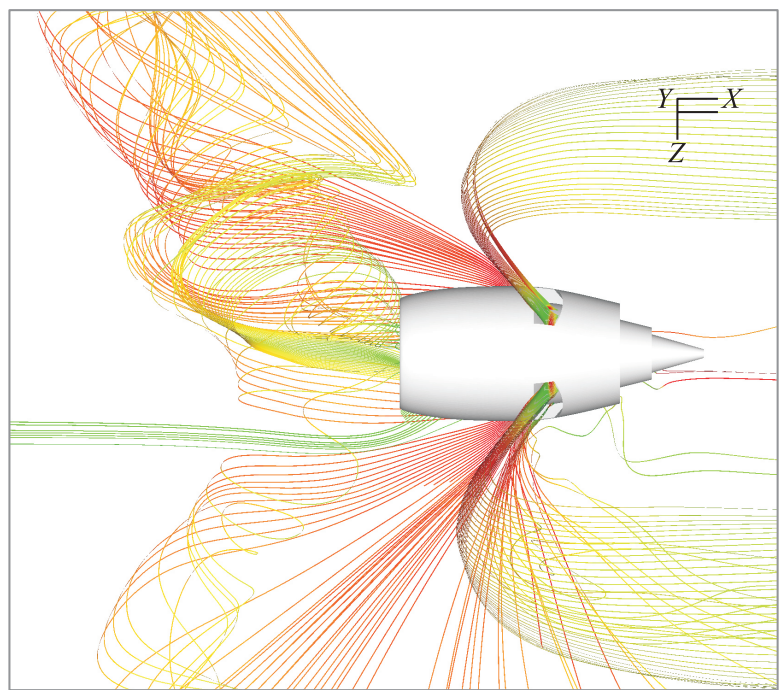

(a)

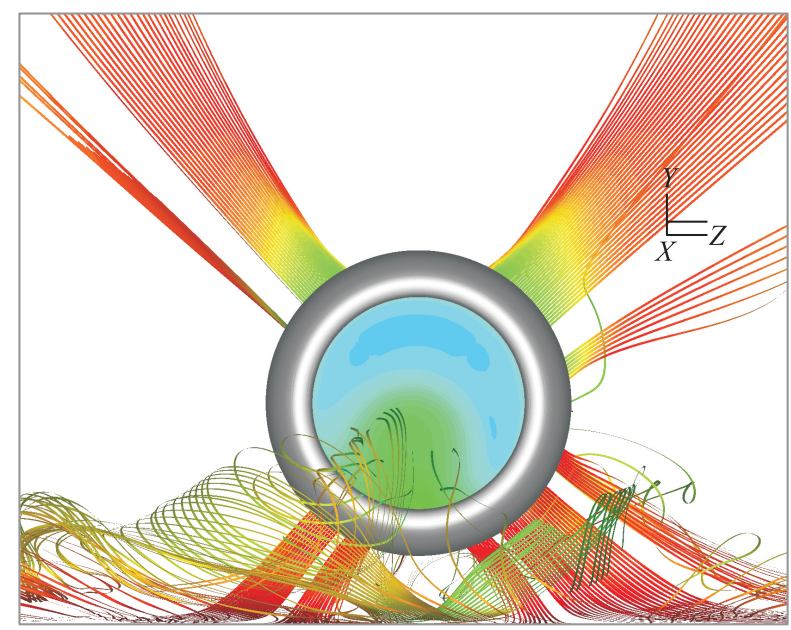

(b)

Figure 3 Streamlines at $u_{\infty}=30 \mathrm{~m} / \mathrm{s}$ 
number of cells, the pod surface was surrounded by an "O-grid." In this case, the scope of the refined cell is located only around the pod and does not extend far from it. The total number of blocks of the developed mesh was 340 .

To increase the spatial resolution in the region of reverse jet propagation, nested grids were used. In some mesh blocks located between the pod and the ground and ahead of the pod, the number of cells was doubled in all directions. The total number of cells was more than 6 million. Three million cells were located in the downward surroundings of the engine.

\subsection{Calculations Results}

For fixed geometry of the calculation domain (see Fig. $2 a$ ) and operation parameters of the engine (see Table 1), several calculations were performed for different airplane velocities.

It was shown that the points where the streamlines become reversed generate a pronounced boundary (Fig. 3), which has a U-shape near the plane of symmetry of the engine. This fact is in good agreement with experimental data [3]. In the case where the airplane velocity $u_{\infty}$ is approximately

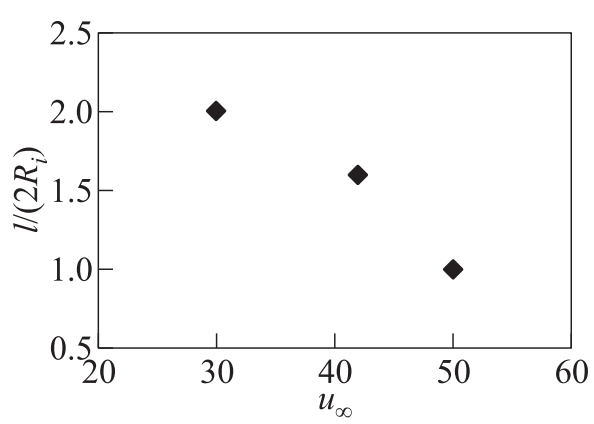

Figure 4 Distance between the engine inlet and reverse jet reversing point $l$ vs. the airplane velocity; $R_{i}$ is the engine inlet radius $50 \mathrm{~m} / \mathrm{s}$, the distance between the engine inlet and the front of the streamline reversal in the direction of the engine axis is close to one diameter. If $u_{\infty} \approx 30 \mathrm{~m} / \mathrm{s}$, then this distance is twice greater (Fig. 4). The colors in Fig. 3 demonstrate the static temperature distribution along the streamlines and in the engine inlet section.

\subsection{Additional Effects Increasing the Particle Ingestion Probability}

The reverse jet during its propagation along the surface has a permanently decreasing axial velocity component. At some instant, this component passes through zero and changes its sign. As was illustrated above, the region of reversed jet reversing creates an uninterruptible boundary moving together with the airplane. The high-pressure reversed jet initiates the motion of sufficiently large particles located on the surface when it is incident onto the surface. Near the symmetry plane, the axial velocity component reaches $90 \%$ of the absolute 
velocity. In the region of reversed jet reversing, this component becomes equal to zero, and the particles seized by the jet return to the surface again. This leads to creation of a zone with an increased particle concentration. Permanent accumulation of particles inside this zone takes place during the airplane motion along the surface. Such accumulation inside the zone moving ahead of the airplane is similar to the bulldozer effect.

It should be noted that the zone with an increased particle concentration passes twice near the air intake during one cycle of the reversed device operation: at the instant of reverser activation the first time and after its deactivation for the second time. The first pass is not that dangerous because the reversed jets do not have enough time to accumulate a large number of particles. In the second pass, the vortex on the air intake appears under the conditions

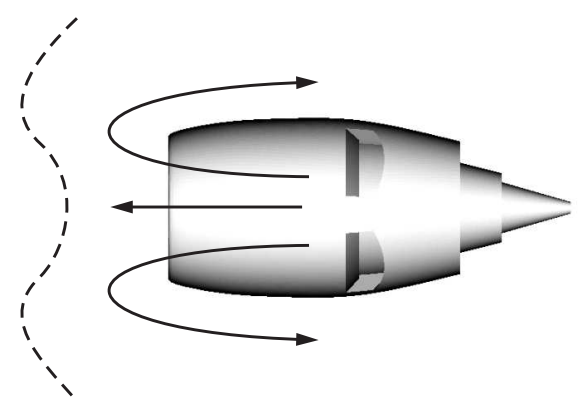

Figure 5 Scheme of reverse jet propagation: solid curve - reverse flow and dashed curve - jet reversing zone where the particle concentration is significantly greater than in other places on the surface. As a result, the particle accumulation time decreases almost to zero, which is needed for creating conditions for ingestion of large particles into the air intake.

The scheme of reverse jet propagation near the surface is illustrated in Fig. 5. The main part of the jet flow propagates in the direction of airplane motion, i. e., the jet propagates in an oppositely directed air flow, which decelerates the jet and reverses it at some distance form the jet source (or from the region of jet incidence onto the surface). In the case of uniform airplane motion and stationary jet outflow, the distance from the jet source to the reversing zone is constant.

The location of the jet reversing zone relative to its source (and other engine elements) can be defined on the base of investigations of jet propagation in the external flow $[4,5]$.

From general considerations, it is possible to say that the jet is rotated when the characteristic velocity of the expanding jet decreases to a value close to the counterflow velocity.

In $[4,5]$, based on experimental data, it was found that the flow reverses the jet when the flow velocity is

$$
u_{\infty} \approx 0.7 u_{m}
$$

where $u_{m}$ is the maximum value of velocity in the jet near the reversed region. 


\begin{tabular}{ccccccc}
\multicolumn{1}{c}{$M$} \\
0.00 & 0.05 & 0.10 & 0.15 & 0.20 & 0.25 & 0.30
\end{tabular}

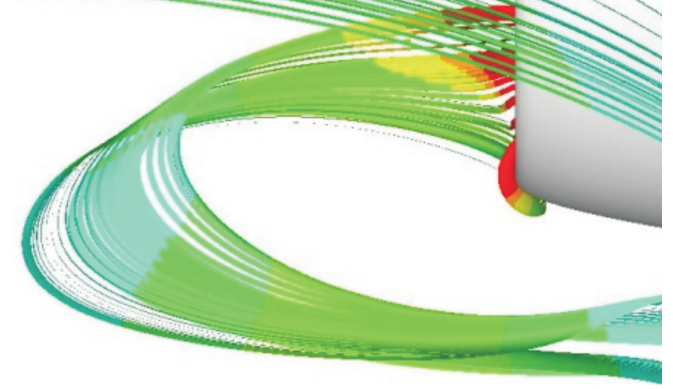

Figure 6 The Mach numbers distribution along the streamlines $\mathrm{M}_{\infty}=0.1$

A similar relationship is demonstrated in Fig. 6, where the reversed jet reversing begins at the Mach number $\mathrm{M} \sim 0.15$ and the counterflow Mach number $\mathrm{M}_{\infty}=0.1$.

The same relations can be derived from the assumption that jet reversing takes place when the value of the impulse flow in the jet with its lateral dimensions $b$ becomes equal to the impulse flow in the part of the counterflow of the same size:

$$
\int_{0}^{b} \rho u^{2} d y=\rho u_{\infty}^{2} b .
$$

The position of the jet relative to the airplane and the engine changes with variations of the jet velocity and the airplane velocity (which is the same as the counterflow velocity $u_{\infty}$ ).

As was mentioned above, the analysis demonstrates that the reverse jet has a large range with reversing activation in the full thrust regime despite a large counterflow velocity. Therefore, the jet reversing zone is located far from the engine air intake (see Figs. 2 and 3). This distance increases as the counterflow velocity decreases. After reverse takeoff or engine regime decreasing, the jet reverse zone appears near the inlet and passes directly under the air intake at a certain time instant. It is obvious that this is a destructive time instant because the reverse jet accumulates particles in this zone during its entire operation mode.

Special calculations of particle trajectories under the conditions of interaction of the near-surface jet with the counterflow were performed to analyze this process. The results of these calculations are illustrated in Fig. 7. The particle 


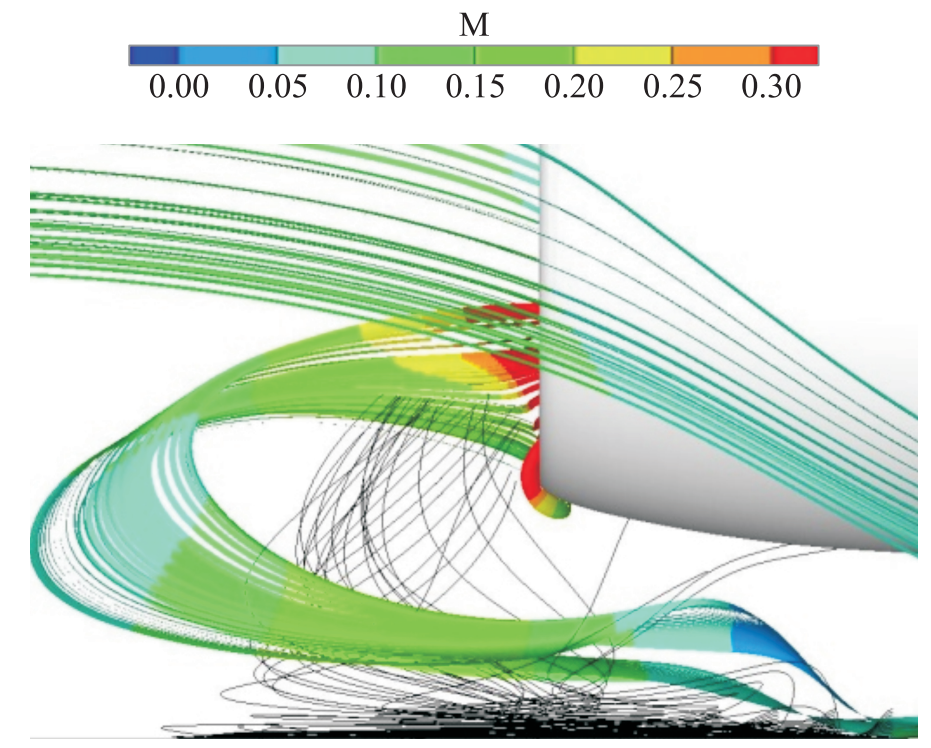

Figure 7 Streamlines (as in Fig. 6) and particle trajectory (black curves)

trajectories in this figure were calculated for arbitrary initial positions. It can be seen that the particles accumulate near the region of jet reversing.

The flow structure demonstrated in Fig. 7 corresponds to the jet reversing zone location near the air intake. The vortex flow captures particles directly from the zone of their accumulation because of the reversed jet action.

The danger of this situation is the effect of enhanced transportation of large particles to the engine inlet, which is caused by presence of a considerable number of particles in the ground part of the ascending flow $[1,2]$. Namely, it is observed when the ascending flow rises from the reversed jet reversing zone.

For suppression of such danger for the engine, a sufficiently intensive jet flow regime is needed for maintaining the reversed jet reversing zone at some distance from the air intake.

Therefore, the decreasing of the reversed regime in correspondence with Eq. (1) must be restricted by the condition

$$
u_{m} \geq \frac{u_{\infty}}{0.7}
$$

which must be satisfied in the near-surface jet flow for the maximum velocities up to some minimum distance $\Delta L$ from the air intake.

In $[4,5]$, a calculation model of the flow in the jet propagating along the surface in a counterflow was described. This model was developed on the base of 


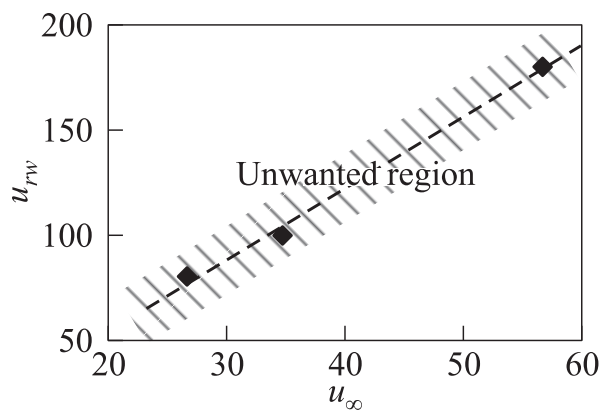

Figure 8 Dependence of the character reversed jet velocity $u_{r w}$ from the counter flow velocity $u_{\infty}$ for its reversing under air intake.

experimental investigations. Based on this model, the distance from the reversed jet source was determined under the assumption that

$$
u_{m}=\frac{u_{\infty}}{0.7}
$$

In the assumption that minimum value of this distance must be correspondent to the distance up to air intake, a value of reversed jet velocity $u_{r w}$ can be determined for each value of counterflow velocity $u_{\infty}$. For safety airplane motion under thrust reversing, it is necessary that for reverse jet velocity $u_{r}$, the conditions $u_{r}>u_{r w}$ were performed. Determined from calculations approximated values of reversed jet velocity $u_{r w}$ for which the reversing zone will be located under air intake for different velocities of counterflow $u_{\infty}$ are illustrated in Fig. 8. The region located near the dashed line corresponds to the unwanted values of velocity $u_{r}$.

\section{CONCLUDING REMARKS}

Based on numerical calculations and experimental results, a qualitative analysis of dangerous effects of thrust reversing devices was performed. Particle accumulation in the zone of reversed jet reversing creates a condition for suction of large particles into the engine. The probability of such a situation appreciably increases if the air intake is located directly above this zone, which takes place when the thrust reversing regime changes to its takeoff. For reduction of this adverse effect, it is necessary that, as the reverse regime is alleviated, the reversed jet velocity should be sufficient for the reversing zone to be located far from the air intake. 


\section{REFERENCES}

1. Krasheninnikov, S. Yu., and D. E. Pudovikov. 2007. Induced flow and ascent of heavy particles when an air intake operates near a surface. Fluid Dyn. 42(4):654-665.

2. Krasheninnikov, S. Yu., and D. E. Pudovikov. 2007. Induced flow and large particles lift at air intake operation near ground surface. EUCASS 2007.

3. Krasheninnikov, S. Yu., and D. E. Pudovikov. 2005. Application of 3D CFD methods to the analysis of large particles ingestion to the engine inlet located near the aerodrome surface. EUCASS 2005.

4. Klestov, Yu. M. 1978. Propagation of a turbulent jet contacting a flat surface in a counterflow. Izv. Akad. Sci. USSR, Mekh. Zhidk. Gaza. 5:56-62.

5. Abramovich, G. N., ed. 1984. Theory of turbulent jets. Moscow: Nauka. 717 p. 